\title{
Major requirements for building Smart Homes in Smart Cities based on Internet of Things technologies
}

Article

Accepted Version

Creative Commons: Attribution-Noncommercial-No Derivative Works 4.0

Hui, T. K. L., Sherratt, R. S. and Diaz Sanchez, D. (2017) Major requirements for building Smart Homes in Smart Cities based on Internet of Things technologies. Future Generation Computer Systems, 76. pp. 358-369. ISSN 0167-739X doi: https://doi.org/10.1016/j.future.2016.10.026 Available at https://centaur.reading.ac.uk/67947/

It is advisable to refer to the publisher's version if you intend to cite from the work. See Guidance on citing.

To link to this article DOI: http://dx.doi.org/10.1016/j.future.2016.10.026

Publisher: Elsevier

All outputs in CentAUR are protected by Intellectual Property Rights law, including copyright law. Copyright and IPR is retained by the creators or other copyright holders. Terms and conditions for use of this material are defined in the End User Agreement.

www.reading.ac.uk/centaur 
Central Archive at the University of Reading

Reading's research outputs online 


\title{
Major Requirements for Building Smart Homes in Smart Cities based on Internet of Things Technologies
}

\author{
Terence. K. L. Huia ${ }^{\mathrm{a}, *}$, R. Simon Sherratta ${ }^{\mathrm{a}}$, Daniel Díaz Sánchez ${ }^{\mathrm{b}}$ \\ ${ }^{a}$ School of Systems Engineering, University of Reading, UK \\ ${ }^{b}$ Dep. Ing. Telemática, Universidad Carlos III de Madrid, Spain
}

\begin{abstract}
The recent boom in the Internet of Things (IoT) will turn Smart Cities and Smart Homes (SH) from hype to reality. SH is the major building block for Smart Cities and have long been a dream for decades, hobbyists in the late 1970s made Home Automation (HA) possible when personal computers started invading home spaces. While SH can share most of the IoT technologies, there are unique characteristics that make SH special. From the result of a recent research survey on SH and IoT technologies, this paper defines the major requirements for building SH. Seven unique requirement recommendations are defined and classified according to the specific quality of the SH building blocks.
\end{abstract}

Keywords: Smart Cities, Smart Home Requirements, Ambient Intelligence, Internet of Things, Home Automation

\section{Introduction}

The term "Internet of Things" first appeared in 1999 when Ashton [1] presented a report on Radio Frequency IDentification (RFID) to Procter and Gamble. The idea of automatic data collection using RFID and sensing technology, together with the continuous development on Wireless Sensor Networks (WSNs), Machine-to-Machine (M2M) architectures, Artificial Intelligence (AI) and semantic technologies have enabled IoT to blossom. Cisco has predicted that 50 billions of Things will be connected to the Internet by 2020 , likely to be 6.58 times more than the estimated world population [2]. Holler et al. [3] pointed out that the major reason for IoT to take off was the need to enable technologies at the right cost.

IoT is considered as an extension of the existing Internet where Human-to-Human $(\mathrm{H} 2 \mathrm{H})$ interaction has dominated the daily network communication. Familiar $\mathrm{H} 2 \mathrm{H}$ examples are text messaging, voice and video conferencing and social networking. Human-to-Machine (H2M) interaction has become another important part of Internet communication when machines get smarter with AI. A smart machine, or intelligent computer server, can tailor make content for a dynamic web page and present it to a particular user according to his/her browsing history. Miniaturisation of electronic components according to Moore's law enables networked computers to be embedded into anything we want. Thus Things are becoming computerised, smart, and connected to the Internet as well. Computers will be everywhere, network connected, and invisibly living with humans: a situation described by Wesier as Ubiquitous Computing over two decades ago [4]. IoT is a concept to get Things connected to the Internet, and Thing-to-Thing or M2M interaction is the core IoT technology. Global scale IoT applications have been found in many areas from domestic to industry and from national to international.

Cyber Physical Systems (CPS) adopting IoT technologies seamlessly integrate physical components with cyber space through contemporary computing and networking technologies. The real-time operation in the physical world dictates the difference of CPS from today's computing and networking abstraction [5]. Typical CPS applications are SH and Ambient Intelligence where the monitoring, controlling and automating functions are accomplished through connected sensors and actuators. Tele-care services support elderly and disabled people to connect to health care

\footnotetext{
${ }^{*}$ Corresponding author

Email addresses: t.k.1.hui@pgr.reading.ac.uk (Terence. K. L. Hui), sherratt@ieee.org (R. Simon Sherratt), dds@it.uc3m.es (Daniel Díaz Sánchez)
} 
monitoring services provided by medical institutions from a distance. Telematics makes driverless vehicles commuting in the cities possible by feeding wireless real-time road traffic information constantly to the built-in car navigation systems.

Smart Grid focuses mainly on energy saving for homes and businesses based on the power grid to collect usage data from appliances. IoT technologies support the algorithms for balancing the power from the power plant and the in-house power sources, e.g. solar panels, and give the users a better decision about energy consumption [6].

Industry has recently embraced IoT technologies to boost productivity. "Industrie 4.0 " is now a popular term in Germany. It describes a vision of the 4th industrial revolution following the three previous generations: mechanisation (Industrie 1.0), Electrification (Industrie 2.0) and Digitialisation (Industrie 3.0) [7]. At the same time, General Electric (GE) has proposed similar vision for US industry by redefining the term "Industrial Internet" which integrates the three elements: Intelligent Machines, Advanced Analytics

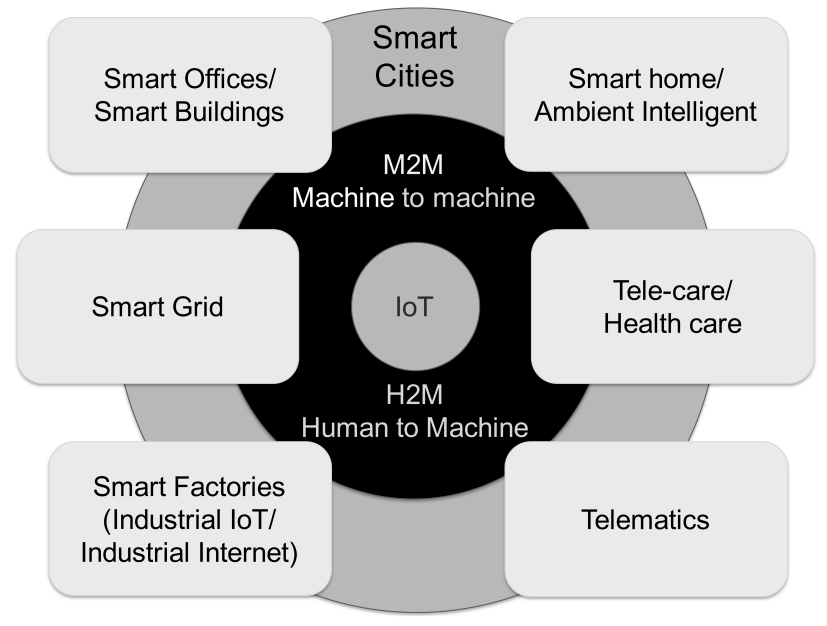

Figure 1: Typical Architecture of a Smart City and People at Work to increase the manufacturing output [8].

Smart Cities apply the technologies into a much wider scale by connecting people in a city to all "Smart Technologies" mentioned above in order to deliver real time information for selected users with correct details at the right time. Figure 1 shows a typical integration of smart technologies in a Smart City architecture.

This paper discusses the latest SH research based on IoT technologies with the objective to classify them into a list of major requirement recommendations for building SH systems. Section 2 describes the latest sensor network technologies and proceeds to list the seven major requirements to build SH systems defined from this work. The challenges we have seen in meeting this requirements are discussed in section 3. Finally a conclusion with future work is presented in section 4.

\section{Smart Home and Smart Cities}

SH is the basic building block for Smart Cities, and the establishment of Smart Cities is a core enabler for the rapid global urbanisation. By 2050, 66\% of the world population will be living in urban areas while the number of "mega-cities" with 10 millions inhabitants or more is expanding in the same pace ${ }^{1}$. A people-centric design approach is adopted in building Smart Cities in order to share resources effectively and intelligently, however, provision of tailor-made services to individual inhabitants is difficult without collecting and learning personal behaviour in public spaces such as smart offices, smart factories, and public transport. SH is the best venue assisting Smart Cities to gain personal data when privacy protection is properly implemented.

SH and HA are used interchangeably in this paper and they refer to the applications of IoT technologies in the home environment. A high degree of heterogeneity makes the connections difficult due to many interfacing technologies available in the market. Things to be connected at home can be low-resource devices such as self-powered lightswitches using energy harvesting as power source, or battery operated temperature sensors which wake up every ten minutes, or even fully equipped multi-core desktop Personal Computers (PCs) running 24-hour per day. Repetitiveness of control processes may vary from 100 times per day down to once per year.

\footnotetext{
${ }^{1}$ http://www.un.org/en/development/desa/news/population/world-urbanization-prospects-2014.html
} 
The story of SH began in the late 1960s when computer amateurs started installing computers at home, and one of the famous home computers at that moment called ECHO-IV was installed by Sutherland for family bookkeeping, inventory taking, as well as house temperature control [9]. When PCs appeared in the mass-market in the late 1970s [10], on-site controlling and automating of home appliances became Do-It-Yourself (DIY) projects by hobbyists. Remote control was achieved by decoding Dual-Tone Multi-Frequency (DTMF) signals through telephone lines when a domestic Internet service was not yet generally available [11]. Research in SH has been progressing but the real adoption is still very low. Greichen [12] described in 1992 the emergence of SH market was "just around the corner" after a decade of research and implementation. Nowadays, after passing another decade from Greichen's work, we have not seen significant SH adoption yet and the high cost, difficult installation and unfriendly operations are still the main obstacles transforming the hype to reality.

\subsection{Sensor Networks for Smart Homes}

The networking of Things such as sensors and actuators is the basic enabler for M2M connectivity. Things can be connected using wired or wireless technologies depending on the home environment. For a wireless connection, short range and long range radio links provide two different communication paradigms to fit for different system architectures.

Wired sensor networks connect Things with fixed wires which are not convenient in terms of installation and extensibility for future upgrade. Wiring may be required to form a home network for transmission of control data, and the network is usually in the form of bus structure such as CEBus, $\mathrm{KNX}^{2}$ and LonWorks. Data transmission over power lines eliminates the needs for running separate physical wires and is good for the retrofit market, Home Plug, Insteon and X10 are typical examples in this category. X10 has been popular in the DIY market since 1975 when Pico Electronics invented the protocol using power lines as data transmission medium [13]. This technology has become a de-facto standard in SH for many years due to its ease-of-use, easy-of-install and easy-of-upgrade by plugging into any power outlet to form the SH net. The notoriously unstable performance due to power line interference prevents X10 to prevail in the SH market, however the high cost-performance-ratio still keeps X10 products in production. Ethernet, except the usage in setting up Local Area Network (LAN) for communication between computers, is also a good candidate for SH connectivity requiring high bandwidth such as high-end audio and video streaming at home. Many wired networks also provide wireless options to cope with the restriction on physical wiring, e.g. Wireless X10 and Wireless KNX.

Bus based $\mathrm{SH}$ technologies such as KNX and LonWorks have not penetrated in the home market except for building management and luxury properties due to their elevated cost but KNX, for instance, is still one of the best choices due to stability and reliability for SH. The major goals of KNX have been detaching the transmission from the control logic and enabling the compatibility among function modules from different manufacturers irrespectively of the underlying transmission technology. KNX relies on the OSI protocol stack and use their own addressing space based on device addresses to interact with a single device and group addresses for joint operation of similar devices. In KNX systems, the function module is governed by a small microprocessor that implements the upper layers and the entire module is plugged in to bus coupler that implements lower layers according to the desired transmission technology that can be low power buses, power line, RF and infrared. The number of devices is limited by the transmission technology and the length of the wires. While IoT for SH opens a number of new possibilities, these buses are interesting especially for HVAC systems and critical infrastructures due to their isolation and reliability. Among the requirements for $\mathrm{SH}$, IoT should provide equivalent security to become an alternative in the context of critical equipment management inside a home environment. Moreover, device interworking, guaranteed in KNX, is still one of the major problems in IoT due to the lack of standards.

A proliferation of ubiquitous Wireless Sensor Network (WSN) protocols has enabled WSNs to dominate the M2M connectivity technology in SH. Low cost, low power, self configurable and expandability are major design criterion for WSNs. Short range radio fits the low cost and low power requirements while mesh networking architectures provide the network nodes to be self-configured dynamically and offer easy expansion. Zigbee ${ }^{3}$ and Z-Wave ${ }^{4}$ are the

\footnotetext{
${ }^{2}$ www.knx.org

${ }^{3}$ www.zigbee.org

${ }^{4}$ www.z-wave.com
} 
most common home control WSN protocols that provide low cost, low power mesh network connectivity. Bluetooth ${ }^{5}$ has long been positioned as a personal area network connecting up to seven surrounding devices not exceeding a maximum of 100 meters (for class 1). With the newly introduction of Bluetooth Low Energy (BLE) 4.1, Bluetooth mesh networking (or Bluetooth Mesh) is possible now to compete with other mesh WSN technologies.

Most wired and short range WSNs require connection hubs or gateways to convert and route internal network data in and out to the Internet. A long range WSN becomes necessary for applications requiring direct wireless connection to the Internet. Cellular networks (e.g. EDGE, 3G, LTE, etc.) provide Internet connectivity for Internet Protocol (IP)-enabled devices, but the connection cost is relatively high. A combination of short-range WSNs and the cellular network is sometimes referred as a Capillary Network which enjoys a complete wireless connectivity for home devices using a cellular connected gateway to exchange WSNs data to the Internet through cellular radio [14]. A new standard proposed by Weightless ${ }^{6}$ using the TV white space as wireless data links enables a cheap alternative $[15,16]$

6LoWPAN ${ }^{7}$, also based on IEEE 802.15.4 like Zigbee and WirelessHart, enables direct IPv6-connectivity to the Internet for resource-limited nodes in WSNs, and its open standard nature supported by IETF (Internet Engineering Task Force) will hopefully make it stand out from the competition. However, different proprietary standards for WSNs are being proposed at the same time from the industry such as WEAVE from Google and HomeKit from Apple, it may take a long time to converge to a globally acceptable protocol standard for SH and IoT.

\subsection{Major requirements for building smart home}

High degree of heterogeneity, low repetitiveness, polarisation of user experience, demands of security and privacy protection, are all typical and critical characteristics of SHs. Human-in-the-loop demand is higher than any other IoT applications since humans are the ultimate owners of all Things in the home space. The complexity escalates when there are multiple owners in a single home space where multiple but different rules must be applied at the same time, in the same place, for the same Things. Yamazaki [17] argued that total automation as the goal of SH technologies was a mistake preventing SH to prevail in the 1970s. Intelligence is becoming a basic ingredient to get automation smarter in SH and IoT technologies.

This paper summarises the major requirements from previous research focusing on SH and IoT. Although SH has been researched and implemented for decades, adoption of SH is still at its infancy. This paper defines the major requirements and proposes the essential elements for building $\mathrm{SH}$ with IoT technologies. While it is believed that more requirements will be revealed in the future when $\mathrm{SH}$ becomes more mature, the major requirements derived from this research are found to be:

1. Heterogeneity

2. Self configurable

3. Extensibility

4. Context Awareness

5. Usability

6. Security and Privacy Protection

7. Intelligence

The following sections now describe each requirement in detail.

\subsubsection{Heterogeneity}

Heterogeneity is the ability to let different types of connected Things exchange information in a given network. Things are typically electronic devices embedded with network-connected computers, they may have different processing power, different input-output facilities, different scale of resources, different connectivity technologies, and different communication protocols.

\footnotetext{
${ }^{5}$ www.bluetooth.org

${ }^{6} \mathrm{http}: / /$ www.weightless.org

${ }^{7}$ https://tools.ietf.org/wg/6lowpan/
} 
Things exchange information easily when they connect with the same connectivity technology inside a given wired or wireless network. When Things are not IP-enabled devices, the connection to the Internet is done through a gateway to translate the non-IP to IP connectivity. The level of heterogeneity increases when two or more networks with different technologies are required to work together. The connection gateway architecture provides a platform so heterogeneous Things can talk to each other in a combined network.

Perumal et al. [18] defined three interoperability levels for heterogeneous systems: (1) Basic Connectivity interoperability concerns more on the physical connections of devices; (2) Network Interoperability describes the data communication management, and (3) Syntactic Interoperability refers mainly on the application level. The authors verified the concept of the home system with a Home Gateway running Simple Object Access Protocol (SOAP) to link up all heterogeneous sub-systems. To simplify the structure, they only used Ethernet to connect all heterogeneous devices running the Transmission Control Protocol (TCP) protocol.

Server Centralised Architecture (SCA) was described by Xuemei and Gang [19] as the approach to connect devices in the home space using a Home Gateway. They proposed that appliances can be divided into device and service layers in order to identify devices as services instead of individual functional interfaces. The authors further proposed a Service Oriented Architecture (SOA) to increase the degree of heterogeneity for some servers in terms of scalability, interoperability and reliability. A network of physical devices becomes a network of services using this approach. Figure 2 shows a typical heterogeneous network for the home.

Ontologies have been widely proposed as a vehicle to reduce the impact of heterogeneity in IoT [20]. These approaches deal with the heterogeneity of IoT components and also the unknown nature of the network topology (and their dynamic nature) by

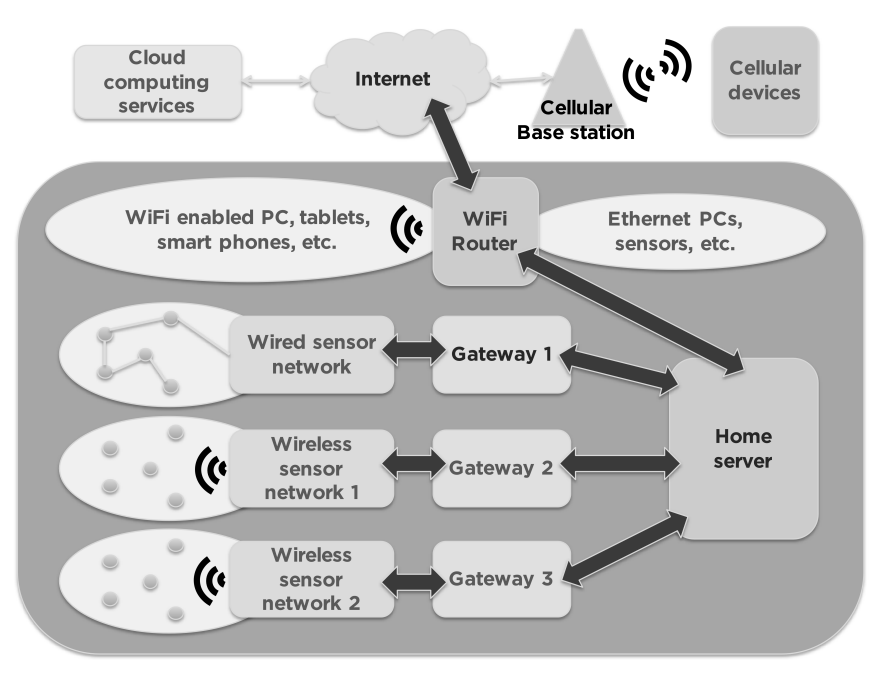

Figure 2: Typical Heterogeneous Home Network modeling a set of ontologies that describe device functionalities. Complementary, others, as [21], model ontologies for describing the communication interface at application level. Several efforts, as [22] focus on orchestrating things around existing ontologies. Even though ontologies are valuable tools for describing things, functions, services and interfaces, and reducing the impact of the heterogeneity, their processing involves a huge resource consumption. The use of ontologies could be embraceable in practice by off-loading their processing to a cloud or a home gateway.

A software framework accommodating all the related services in a home network becomes a convenient and efficient way to establish a network of Things. Open Standards Gateway initiative (OSGi) ${ }^{8}$ started from set top box design provides an open specification based on SOA for setting up a framework using Java technology. Middleware runs on top of the framework to exchange information between individual service. Ready made Middleware can be found that works seamlessly with other user applications in the same framework. A typical example is a service discovery application, e.g. SLP (Service Location Protocol) or UPnP (Universal Plug and Play), which is useful to locate services in a heterogeneous network. Maternaghan and Turner [23] proposed a Tele-care System based on OSGi framework, they also turned the research project into a product called Homer which linked up components and services in an OSGi enabled home server. Homer provided control and automation algorithms to all connected components through its own Application Programming Interface (API) which may be an obstacle for general adoption by various manufacturers.

\footnotetext{
${ }^{8}$ http://www.osgi.org
} 
A combination of Middlewares and gateways has been dominating the home server architecture for recent years. An obvious offloading of the home server is happening with the proliferation of cloud computing services and the popularity of high speed home broadband. Simple gateways performing protocol conversion is enough to connect Things in WSNs to the Internet through the cloud. Dealing with complex compound services within the home environment and the cloud has triggered the development of scalable technologies that transfer partially the data center cloud technology to the home environment or federations of users equipment. Some works such as [24], [25] and [26] proposed middleware for Set-top boxes and gateways for classifying, searching, and delivering media inside home network and across the cloud that interoperates with several home protocols. Others went a step beyond providing also frameworks for distributing applications [27] or accessing secure home services from remote locations transparently without breaking home protocols. All these movements have triggered the concept of fog computing $[28,29]$ that basically defines a new set of devices, called Fog devices, between the device and the cloud, that can absorb partially the load that would be otherwise delivered to the cloud leveraging resources that would be misused and reducing the exposure of private data.

Services from many cloud computing suppliers provide connection with XaaS (Everything As A Service) for remote monitoring, controlling and automating Things in the SH sector. Soliman et al. [30] demonstrated a home network to the cloud based on SaaS (Software As A Service) and PaaS (Platform As A Service). Zigbee devices were connected to the cloud though a simple Arduino-embedded gateway, and all management and security services were provided in the cloud through common web services. Heterogeneity is achieved with the plethora of contemporary home management services from the cloud computing suppliers. Applications in IoT generate huge amount of information worth to be processed. To facilitate interaction with the cloud, manufactures are developing small general purpose boards with a built-in operating system, libraries and the software development kit for connecting things to their cloud services, providing so a customizable solution for IoT in a single bundle. Despite off-loading to the cloud complements IoT local applications with several other services, it also introduces a major problems as data lock-in. IoT frameworks for SH should support alternatives to current closed manufacturers' clouds like actor or data flow models in which applications can be distributed to and instantiated in several different locations and orchestrated in a simple way using asynchronous messaging.

Aligned with the latter, it would be necessary to connect manufacturers and developers to businesses and consumers in a standardized way as proposed by the COMPOSE project in their market place for the IoT ${ }^{9}$.

\subsubsection{Self Configurable}

Self-configurable refers to the capability to add and remove Things in SH networks automatically through altering the context of the Things or the network topology. Things in SH are sometimes non-permanent residents, such as consumables (e.g. a light bulb, an ink cartridge, etc.), or movables (e.g. a mobile heater, a trolley, etc.), or wearables (e.g. a smart watch, a heat rate monitor, etc.) and they may come and go from time to time. The registration and reregistration processes should be done quietly and autonomously without user intervention. Self-installation for brand new Things in SH contributes to another measurement for this requirement. New technologies from the market or

\footnotetext{
${ }^{9}$ http://www.compose-project.eu/
} 
new requirements from the home owners usually gain new members to the existing SH network, an easy installation helps the market grow. First time installation of SH systems normally requires professionals. Easy setup process or auto-setup will be the ultimate goal for non-technical users when SH technology becomes more mature.

Existing mesh WSNs already provide self-configuration algorithm to recover the network operation when there are defective nodes or broken paths blocking the data flow in a network. Hwang [31] pointed out that the simple self-configuration and self-healing mechanism provided by Zigbee specification is too slow. The low power nature of Zigbee network prevents the nodes from frequent wake up and the detection of a Beacon Lost will be slow to confirm the loss of synchronisation. An orphan scan initiated by the loss confirmation will then renew and recover the routing according to a preset response time. An enhanced method was proposed by Hwang to speed up the self-configuration and self-healing processes, as well as to improve the orphan propagation problem from the user application layer.

There are other popular mesh WSN technologies available in the market supporting self-configuration and selfhealing mechanism such as Z-Wave, WirelessHart ${ }^{10}$, etc. An interesting alternative is Bluetooth BLE (or Bluetooth Smart) which also supports mesh networking with a scatternet topology. Scatternet combines many piconets and the master of each piconet becomes slave of adjacent piconet, thus this combination turns many single hop personal area networks into multi-hop mesh network. Scatternet was defined together with piconet as the network topologies in early versions of Bluetooth specification and some research has already proposed methods implementing mesh networks based on scatternet specification [32]. ABI Research's forecast of 10 billions Bluetooth-enabled devices in the market by 2018 puts Bluetooth back under the spotlight [33]. Bluetooth SIG has recently setup the Bluetooth Smart Mesh Working Group to promote the technology for IoT.

There is still very little research on auto-setup for SH up to now, only methods on easy configuration or easy setup are found. An earlier attempt by Leeb et al. [34] back in 1996 presented a configuration tool called Homenet for configuring home appliances using a Graphical User Interface (GUI) on a computer console. Their idea relied on the fact that users usually added appliances one by one so users became the installer all the time. Each appliance was represented as a list of functions and users configured the appliances by combining functions of different appliances to form an object. The procedure looks intuitive and easy to operate but the prerequisite is the provision of the list of functions from appliance manufacturers.

In a different approach, Chen and Chang [35] promoted expert assistance from installers and designers through cloud services. An interaction based on cloud computing links up users, designers, manufacturers and installers together to complete a tailor-made SH system. SaaS combines different applications in cloud computing and achieves the goal from drafting user requirements, to setting proposals, to selecting products all in the shared Computer Aided Design (CAD) applications. Users are heavily involved at the beginning to define the requirements and all technical details are hidden. When the platform grows with more designers and manufactures, the burden of users as installers can be offloaded to selected experts.

Other approaches, more related with WSN but applicable to the IoT SH, have developed SensorML as a lightweight markup language to describe sensors and actuators in a very simple way [36] ${ }^{11}$. SensorML does not provide the richness of ontologies when it comes to define Things in general but it may have an important role to play in the context of IoT for SH since many device manufacturers may find the language expressive enough for small or single purpose sensors and actuators.

\subsubsection{Extensibility}

Extensibility is the capability of a SH system to extend the functions or configurations of the connected Things, the scale of the network, and the adoption of new technologies.

WSN is always a better choice over wired network for SH networking in terms of devices upgradability and network scalability. Data over power-lines improves the extensibility in certain extent but the bandwidth is normally not enough for upgrading the functions of connected devices. Over-the-air (OTA) software update has long been a feature for many WSN technologies, including Zigbee, Z-Wave, 6LoWPAN, BLE and many other WSN protocols. Brown and Screenan [37] studied the different techniques for software updating in WSNs, and the possible problems affecting the reliability of code transfer mechanism. Autonomic software and configuration upgrade is a critical feature

\footnotetext{
${ }^{10} \mathrm{http}: / /$ en.hartcomm.org

${ }^{11} \mathrm{http}: / /$ www.opengeospatial.org/standards/sensorml
} 
for WSNs as a post-deployment strategy, deficiency in the current technology in terms of updating management on error handling, feedback and configuration should reveal more research in the coming future.

Extending the scale of the network is also a built-in feature for most of the mesh network WSN architectures. Adding or removing a device in a mesh network activates the reset of a routing table which requires intelligent algorithms to avoid a slow response due to the infrequent wake up time for power-constrained network nodes as we have discussed in section 2.2.2.

A good example of modular design in a form of middleware running on OSGi framework was demonstrated by Huang et al. [38]. All modules were upgradable and new modules could be added as long as they used the same interfacing standard. The authors reviewed the difficulty when the same modules were working loosely without a common standard mechanism. An alternative common platform is available from the recent boom on cloud computing which provides an API for users to configure the services through a simple web service protocol using web browsers.

As mentioned before, the actor model can complement SH [39] with pretty good extensibility. The actor model is a well-known mathematical model of concurrent large scale computation. Actors are the universal primitives of computation being actors small single threaded applications with a mailbox and some state. The actor behavior is controlled by the internal state and the messages received. Since actors run isolated from each other it can help controlling the resources it consumes whereas enable the deployment of a number of other new functionalities to an existing device just adding new actors remotely.

\subsubsection{Context Awareness}

Context Awareness concerns the capability to detect and react when a Thing itself is changed (e.g. it is moved to a different location, or its property is altered, etc), or the surrounding environment gets changed (e.g. new Things or services are added or removed from the surrounding, etc.).

The concept of Context-Aware Computing was proposed by Schilit et al. in 1994. The authors claimed that the three important aspects of context were: "where you are, who you are with, and what resources are nearby" [40]. Proliferation of mobile computers created the new Mobile Distributed Computing paradigm when mobile met with stationary computing devices. Context detection of the mobile device enables the provision of timely, accurate and relevant services in ubiquitous computing. A simple IF-THEN rule activates proper actions when context is changed, but a more advanced algorithm, or even intelligence is needed when there are multiple context changes in the same environment. The major problem encountered by the authors was the provision of a timely and accurate reaction on contextual information.

Context-Aware technology provides a useful tool to the business world, especially the cellular business. Knowing the location of users enables an effective information push to the users' mobile devices, such as local time and weather, or sales promotion details in a shopping mall, or the direction to the nearest vacant parking spots. In SH environment, context-aware applications initiate a reconfiguration of services according to the context of the mobile Things. For example: a person carrying a bluetooth enabled cellphone is detected by a sensor node when entering a room, the sensing is followed by an authentication service through the credentials registered in the cellphone to confirm a valid entry, a lighting control service is activated to first consult a home management service on the preferred light intensity for that particular person, it then sets the dimmer to the required level and turns on the lights in that room, a new video content has been bought by another member of the room and is set as shared to everyone, so the media management service initiates a message service to push a message to the cellphone to alert the new addition of video content, the person responses to the message and confirms a play action, the media management service then proceeds to play the movie after configuring the TV, video player and audio amplifier. Context-Aware technology is literally the groundwork for building intelligent home.

Activity Recognition has long been an active research topic in Computer Science for decades where human activities are recognised and predicted by classifying those feature-extracted data from passive and active sensors. Probabilistic models such as Hidden Markov Model (HMM), Fuzzy logics, Bayesian Networks and many others are useful mathematical modelling tools acting as classifiers to cope with the complex and noisy sensing environment in ambient intelligence [41]. Human's context can thus be accurately detected from Activity Recognition which has become an important component in Context-Aware systems.

A practical implementation of Context-Aware Home called the Ubiquitous Home was setup as test bed by Yamazaki [42]. Ubiquitous Home differed from other SH experiments by implementing stationary monitoring and sensing devices such as cameras, microphones, floor and infra-red sensors at every corner of the home space together 
with a movable robot to collect both passive and active data. Middleware communicated between servers and databases so as to configure corresponding services according to the context. This was a large scale deployment of sensors in a real home space to verify context-aware SH technologies, and the setup helped collect data for analysis. For real life applications, the use of cameras to detect context may be argumentative in privacy protection.

$\mathrm{Ha}$ and Byun [43] proposed another setup to showcase Context Awareness using wearable sensors in a homecare environment. A wearable computer in the form of a wrist-watch contained 3-axis accelerometer to detect user's motion, and a built-in radio connecting to Zigbee network provided localisation data based on radio fingerprinting method. All services (e.g. 3D motion detection service, user localisation management service, interface and activation services, etc.) presented as software bundles on an OSGi framework communicated with each other to provide a fall detection system. Recognising human activity such as drinking and eating can be detected through body sound using wearable sensors [44] instead of biometric sensors being directly contacted with human's nervous systems.

Other than location recognition, emotion recognition based on affective computing is also an interesting field catching a lot of researchers' attention under the umbrella of Activity Recognition. Emotional context can be extracted through vision-based recognition that detects facial abstraction and human's posture through depth camera [45], or audio-based recognition where affective features are derived from the pitch, energy, amplitude and formant of recorded segments [46].

There is always a conflict between Privacy Awareness and Context Awareness. User intervention to balance the two could be a way out but the result will be a downgrade of autonomy. Further research on balancing these two requirements will be necessary in the coming future.

\subsubsection{Usability}

Usability encompasses the quality of easy to use and easy to learn for non-technical users in a given SH system. Since all Things in a SH are embedded computing devices, the requirement of usability in SH is closely related with the technologies and theory of Human Computer Interaction (HCI).

Technological advancement should aim to improve the quality of human life and the usability of new technology plays a vital role for its success. Usability engineering, User Experience (UX) and many other topics in HCI are major studies to improve the usability of technologies. A user-friendly interface has always been the design goal. A failure in synchronisation between technological advancement and the User Interface (UI) development can turn user-friendliness into user-unfriendliness. Corn [47] pointed out that people were tethered to the technology treadmill, interacting with technology became a daily activity. Nowadays SH and IoT are major technologies that tether heavily with humans more than anything else.

International standards for HCI and usability have been developed for many years and only a few of them are dedicated for homes. Evaluation of the usability for a given SH system according to standards is necessary to keep the SH development to the right direction. Moller et al. [48] described how the recent drafted guideline from VDE/ITG ${ }^{12}$ applies to the usability evaluation of SH environments. The guideline reviews the differentiation between the "easy of use" for usage without error, and the "joy of use" for positive consciousness on the user experience. The common and critical aspects of SH environments are proposed and the corresponding services for evaluation are also suggested. This guideline, according to the authors can be used as a basis for SH system evaluation, as well as a provision for future research questions since the $\mathrm{SH}$ technology is far from mature.

Vazquer and Kastner [49] evaluated usability of SH from an opposite direction, they revealed a self-checking algorithm to detect how users were dissatisfied and disagreed with a given SH system. A shadow system added a "shadow" process to every "normal" process in a given SH system, and the purpose was to validate the performance of the corresponding normal processes. The shadow processes detected the level of dissatisfaction and proposed warnings or adjustment to the normal processes, the settings would be reconfigured automatically or based on user involvement. This system does not provide a guideline for initial system implementation but provides a close loop feedback for improving usability autonomously.

In-situ operations for SH are usually rely on console type interface encompassing displays (e.g. computer monitor, projector, or TV, etc.) and pointing devices (e.g. keyboard and mouse, touch screen, infrared remote controller, etc.). Web services provide convenient ways to support the communication for all basic user interactions through TV with

\footnotetext{
${ }^{12}$ http://www.vde.com/en/Technical Societies/Pages/ITG.aspx
} 
dedicated remote controllers [50]. Some researchers repackage computer consoles into a form of electronic mirrors using flat panel displays embedded with cameras, microphones, touch inputs as well as other sensors to keep the computer less visible at home [51]. Transforming UI to mobile devices increases the mobility but the consideration to fit in all necessary controls in small screen requires special configuration [52]. Natural User Interface (NUI) is adopted by many researchers as a method for SH interaction to meet the easy to learn criteria. Voice activation becomes a natural way to communicate with the SH systems, but the voice sensing facility must be good enough to capture voice from anywhere or at least most areas in homes. A microphone array as the front-end of a vocal interface at home was proposed by Coelho et al. [53]. Augmented Reality (AR) provides a more intuitive way for human interaction by augmenting digital information onto the images of the home environment captured by fixed cameras or cameras on mobile phones. A user can make an informed decision according to the augmented information on top of the image [54]. Gesture detection enables elderly and disabled people to interact with SH system through some AI algorithms, so users only post some simple gestures in front of cameras in order to perform predefined actions [55]. Gesture detection is a very broad subject which needs in-depth research when the technology applies to all types of people, more research are found in [56]. Brain Computer Interface (BCI) has been getting a lots of attention in HCI research which detects Electroencephalographic signals (EEG) to control computer devices. Applying a BCI in the $\mathrm{SH}$ is becoming obvious as an alternative to gesture detection, and a BCI can be used by people with very limited body movements at home [57]. Finally, we close this section by introducing social networking as a UI for the SH. Popular social networks such as Facebook, Twitter, Google+ provide the necessary services to link up Things as separate entities. Since Things in SH are already treated as services which can be registered in the social network as entities utilising all facilities supporting by the social network platform, then when there is a change in the Things at home, an alert can be sent to the users if the Things are treated as "friends" of the users' personal groups [58].

Usability engineering has long been a core topic in HCI criticising the effectiveness, efficiency and satisfaction of any UI design, and recently UX treated by many people as the extension of usability has also been catching a lot of attentions. ISO9241 proposes the definitions for both "usability" and "UX" but there is no formal definition on the relationship between the two terms [59]. Achieving the goals with satisfaction seems to be the major concern for usability, and the measurement of humans' behaviours contributes to the UX quality [60]. Enhancing usability can also be done through human behaviour prediction thus an aligned system response or a list of choices will be given to users according to the prediction result [61]. As we have discussed in 2.2.4, Activity Recognition is a current research topic using mathematical models to predict human behaviours based on input collection from passive data such as RFID [62] or dynamic audio/video based content [63]. Promising results were shown from various prediction methods such as Artificial Neural Network [64], Affective Computing [65], big data algorithm [66] and deep learning algorithm [67].

More research is needed to find the right approach to interact humans with the SH system, with the objective for a high degree of usability. Standards for usability are critical for the whole IoT for SH paradigm, and a cross-platform collaboration between engineering science, behavioural science and psychological research is clearly unavoidable.

\subsubsection{Security and Privacy Protection}

Security and privacy concern the level of protection against malicious attacks and any unauthorised use of private information and they have always been a huge challenge in cyberspace. Stealing confidential materials from business servers, personal photos from private clouds, video content from IP-connected home cameras are typical examples of Internet hackers breaking security. Sharing personal shopping habits, revealing the whereabouts of people and exposing personal details to unauthorised third parties are common behaviours affecting privacy. SH will definitely amplify the impact of the challenge by multiplying the numbers of connected devices and services to the Internet. While link-level security is useful, end to end security is most desirable.

Islam et al. [68] claimed that security protection had to be embedded into each node of WSN since every node could be a target for security attack. A list of requirements for a secured systems was revealed: Confidentiality, Integrity, Freshness, Availability and Authenticity. Cryptographic techniques were explained as the key technology for security protection along with the descriptions of the different types of security attacks with suggested solutions. The authors also divided Privacy into Data-oriented and Context-oriented which corresponded to the attack of the data content itself by internal or external adversaries, and the attack of the locations or timing of the data collection respectively. 
Although most of the existing WSN technologies claim to provide certain security mechanisms, systems integrating contemporary WSN technologies may have vulnerable points not visible or obvious with individual technology for easy security attacks. The leaks from the weakness of each communication technology involved in a complete SH system add up to a new picture of security threats. A real life example showing the vulnerability of a SH network using WiFi protocol for data communication and DECT (Digital Enhanced Cordless Telecommunications) protocol for digital voice communication was demonstrated by Sanchez et al. [69]. The authors revealed the easy detection of B-Field and PP frames from DECT transmission packets when even the latest encryption was implemented. This information supplied context-aware information to attackers, for example, when and where a call was made and for how long. A simple setup using a sniffer near the radio coverage of a WiFi network can capture the MAC addresses and analyse the patterns of the encrypted data packets in order to make a smart guess on web sites visited. New strategies for security and privacy protection are therefore necessary to protect the system as a whole entity by adding all threats from individual technology.

Modern identity technologies (IdM) have been of paramount importance in enabling complex services across a single or multiple domains. Within modern identity approaches, the federated identity and the user-centric models are the most successful ones. Both of them have peculiarities that make their adoption suitable for IoT SH [70]. Federated identity requires human intervention for setting up a circle of trust (CoT) around an identity provider. Services should be registered in that CoT in order to fetch identity information about clients. User-centric identity, where user can be either a real user or a service, is more flexible that federations since the user can combine identity provider credentials (subject to verification) with asserted preferences. In both cases, the identity conveyed to services (or devices providing a service in the context of IoT) is a set of attributes that can be standard or application specific. On top of these identity technologies, other traditional services can be built as authentication, authorization, access control. Due to the heterogeneity present in IoT environments, this kind of security solutions contribute not only providing security services but also serving to other purposes as service discovery and preference assertion.

Nevertheless, state of the art identity systems as SAML (Security Assertion Markup Language) [71] or Info Cards, are way too complex for small devices. Further research on adapting these systems to IoT environments should be conducted.

The cornerstone of security systems is trust. IdM systems manage trust in different ways as stated in [72], but it is always handled in a very static fashion. For instance, SAML and Shibboleth employ pre-existing trust relationship usually based on PKI (Public Key Infrastructure) so a federation implies the aggregation of large lists of providers that agree to use common rules and contracts being hard to deploy and maintain, and high dependence on central authorities [73]. OpenID considered initially a trust-all-comers model but newer versions are dealing with trust establishment. IoT environments need to overcome that trust staticity allowing new things to be added to the ecosystem and allowing new users to interact (securely) with them. To accomplish that trust management and establishment there are two different approaches to be considered trust management and trust negotiation.

IoT SH is and will be a high dynamic ecosystem with a high rate of replacement and new comers, so is worth to handle trust management dynamically by mimicking humans behavior so considering the history of interactions, the context, and the scope to derive trust levels for every request $[74,75]$. Moreover, to enable new things and users to be seamlessly incorporated into the system, fair Peer-to-Peer (P2P) trust negotiation schemas are necessary [76, 77]. This kind of trust negotiation systems are able to authenticate and authorize estrangers, relying on the fact that any resource is protected by a policy that expresses which credential(s) should be disclosed to obtain access to it. Other works, as [78], successfully propose dynamic identity federation systems based on trust management and risk assessment.

Finally, when it comes to the user protection, one of the most worrying problems of IoT is privacy especially when devices are off-loading tasks to the cloud. Letting a device to transfer personal information as habits, media or preferences requires user consent. That user consent is considered granted upon user acceptance of the service level agreement or license bound to the device. The problem is that effectively analysing agreements and contracts could be overwhelming for average users and personal information may have a second life on the internet even after the device is no longer used. Current security framework, even the most sophisticated ones as IdM systems, are not ready to cope with user consent revocation so users may lose control over the use and flow of their personal information [79]. Revoking consent is the ability to grant or withdraw consent of specific actions over data to certain entities and is part of the privacy rules described by the OECD (Organization for Economic Cooperation and Developments) principles and GLBA (GrammLeachBliley Act), and the COPPA (Childrens Online Privacy Protection Act). IoT solutions for SH should cope with user consent whenever user data is transferred outside the boundaries of the home environment. 


\subsubsection{Intelligence}

Intelligence in $\mathrm{SH}$ is the ability to predict human behaviour from the collection of raw data, the management of information, the learning of past experience, the understanding of the surroundings, and the adaptation to dynamic environments. The definition derived from the concepts of Knowledge Management and the meaning of Human Intelligence is "Human Intelligence, mental quality that consists of the abilities to learn from experience, adapt to new situations, understand and handle abstract concepts, and use knowledge to manipulate one's environment"13.

Data Flood is a big challenge when static web pages migrate to dynamic in the web 2.0 revolution where user-generated content contributes to additional data sources. IBM ${ }^{14}$ reviews that 2.5 quintillion bytes of data are created everyday and IDC ${ }^{15}$ also shows that the digital universe is growing 40 percent a year. The challenge may rely on good management and intelligent use of huge amounts of data generated from IoT. AI, semantic reasoning and other semantic technologies will help transform data into knowledge and some researchers in these areas are making significant progress. Although recent research, such as deep-learning, has shown promising results to get machine intelligence closer or even surpasses humans in certain areas, the final intelligent actions based on derived knowledge still relies on humans in a human-centric technology like SH.

Recent research on building intelligent SH systems comes towards SOA where devices become services though abstraction which wraps device functionality into machine-understandable format. A typical SOA for intelligent SH system is depicted in Figure 4 which shows how middleware technology interoperates with various services from heterogeneous connected devices. Middleware is usually deployed as a MulitAgent System (MAS) with each software agent performing unique interoperation between services autonomously inside intelligent system platforms [80, 81].

Connected Things are usually deconstructed into services in terms of individual function or group of functions from a particular Thing or an integration of Things through a Device Management Agent. Chin et al. [82] introduced a concept of decomposing
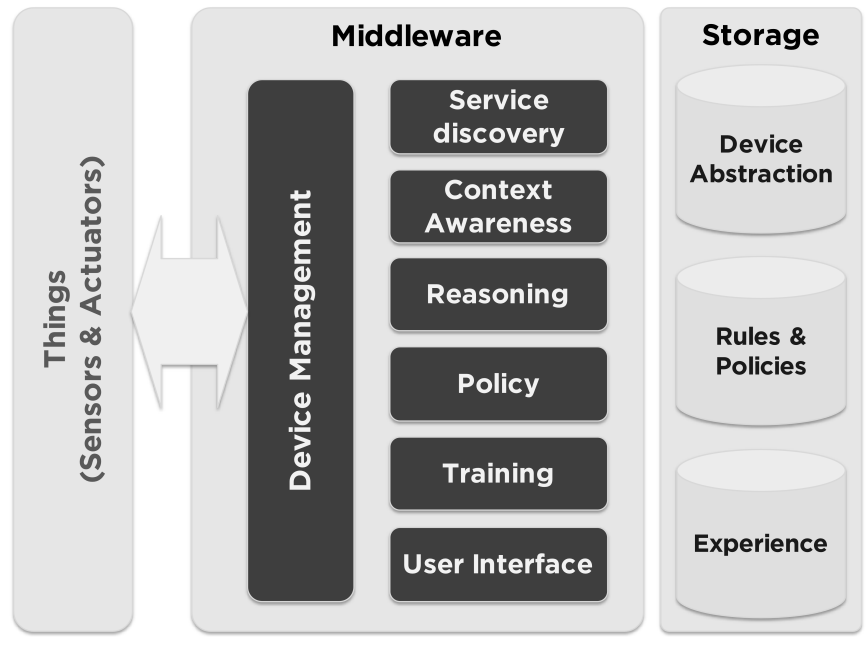

Figure 4: Typical SOA for intelligent SH system appliances into atomic network services using Ontology description language, and a new appliance became Meta-Appliance (MAp) which integrated functions from different appliances and other software applications. Context-aware agents kept track of the context for each service and make sure the corresponding service was available when other agents requested. The UI agent used a goal-driven input from users or other services as requirements and activated the service management agent to meet the goal. Each service or device abstraction was registered as a machine readable entity using Ontology based description languages so service management agent could initiate semantic reasoning and take actions according to predefined policies. Policies were normally based on AI tools such as Artificial Neural Network (ANN), Bayesian Network, etc. Decisions were presented as automation or feedback through user interface, at the same time the results were archived as input for machine learning agent to get the system much smarter through training from past experience. Fabbricatore et al. [83] demonstrated how reasoning, learning and user feedback could work together in a close-loop intelligent resource management framework.

An interesting idea to push all intelligent services to the cloud was found from Chen et al. [84]. The authors added one more member to the XaaS with WaaS (Wisdom as a Service) which implemented the DIKW (Data-Information-

\footnotetext{
${ }^{13}$ http://www.britannica.com/EBchecked/topic/289766/human-intelligence

${ }^{14}$ http://www-01.ibm.com/software/data/bigdata/what-is-big-data.html

${ }^{15} \mathrm{http}: / /$ www.emc.com/leadership/digital-universe/2014iview/executive-summary.htm
} 
Knowledge-Wisdom) hierarchy on a cloud computing platform. Local captured data was forwarded to the cloud services which provided all necessary middleware and intelligent services. Results were sent back to users through an Internet connection. Resources in a central shared architecture over the cloud are much larger than an individual SH system, but the response time, connection robustness, and security remain major concerns.

Referring again to section 2.2.5, the prediction of humans behaviours using activity recognition becomes a key enabler for ambient intelligence, and user preferences and habit tracking is among the most important tasks in SH. User habits tracking in SH may benefit from TV and media recommendation systems and should explore these solutions to improve user experience. Solutions are typically classified in two categories attending to the recommendation mechanisms [85] that are content based filtering and collaborative filtering. The first uses the past experience to propose new actions and the second tries to find similar profiles to determine the most probable desired actions. Many other hybrid approaches allows combining both categories. However, there are other problems to be addressed in SH that are not present in other systems as individual identification, minor detection, group preference modeling, guest detection and presence detection that should be further developed. Moreover, this aligns to security and access control and trust management for addressing guests requests.

\subsubsection{Summary}

This paper reviews the application of major contemporary IoT technologies to building SH and we have classified them according to the proposed requirements. Overlapping is found where one technology can help building SH and meet multiple requirements, thus a cross reference chart is used here to clarify the relationship. Table 1 provides a summary of requirements dependence on different technologies.

Table 1: Dependency of requirements to technologies

\begin{tabular}{|c|c|c|c|c|c|c|c|}
\hline $\begin{array}{l}\text { Technologies } \\
\text { Dependence }\end{array}$ & Heterogeneity & $\begin{array}{c}\text { Self } \\
\text { Configurable }\end{array}$ & Extensibility & $\begin{array}{c}\text { Context } \\
\text { Awareness }\end{array}$ & Usability & $\begin{array}{c}\text { Security and } \\
\text { Privacy }\end{array}$ & Intelligence \\
\hline $\begin{array}{l}\text { Wired sensor networks } \\
\text { Wireless sensor networks (WSNs) } \\
\text { Mesh networking } \\
\text { Indoor localisation } \\
\text { Wed of Things (WoT) } \\
\text { Service Oriented Architecture (SOA) } \\
\text { Cloud computing } \\
\text { Reactive programming } \\
\text { Human Computing Interaction (HCI) } \\
\text { Activity recognition } \\
\text { Identity Management (IdM) } \\
\text { Artificial intelligence (AI) } \\
\text { Recommendation methods }\end{array}$ & $\begin{array}{c}\sqrt{ } \\
\checkmark \checkmark \\
\checkmark \\
x \\
\checkmark \checkmark \\
\checkmark \checkmark \\
\checkmark \\
\checkmark \checkmark \\
x \\
x \\
x \\
x \\
x \\
x\end{array}$ & $\begin{array}{l}\checkmark \\
\checkmark \checkmark \\
\checkmark \checkmark \\
x \\
x \\
\checkmark \checkmark \\
\checkmark \\
x \\
x \\
x \\
x \\
x \\
x \\
x\end{array}$ & $\begin{array}{c}\checkmark \\
\checkmark \checkmark \\
\checkmark \checkmark \\
x \\
x \\
\checkmark \checkmark \\
\checkmark \checkmark \\
\checkmark \checkmark \\
x \\
x \\
x \\
x \\
x \\
x\end{array}$ & 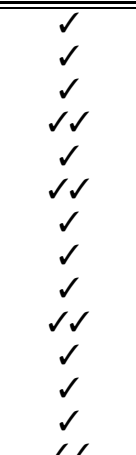 & $\begin{array}{c} \\
\checkmark \\
\checkmark \\
\checkmark \\
\checkmark \\
\checkmark \checkmark \\
\checkmark \checkmark \\
\checkmark \\
\checkmark \checkmark \\
\checkmark \checkmark \\
\checkmark \\
\checkmark \checkmark \\
\checkmark \checkmark \\
\end{array}$ & $\begin{array}{c}d \\
\checkmark \\
\checkmark \\
\checkmark \\
\checkmark \\
\checkmark \checkmark \\
\checkmark \checkmark \\
\checkmark \\
\checkmark \\
\checkmark \\
\checkmark \\
\checkmark \\
\checkmark \\
\checkmark \\
\end{array}$ & $\begin{array}{c}x \\
x \\
x \\
\checkmark \\
\checkmark \\
\checkmark \checkmark \\
\checkmark \checkmark \\
x \\
\checkmark \\
\checkmark \checkmark \\
\checkmark \\
\checkmark \checkmark \\
\checkmark \checkmark \\
\mathcal{J}\end{array}$ \\
\hline $\begin{array}{l}\text { Legend: } \boldsymbol{X}=\text { independent; } \boldsymbol{V}=\text { least de } \\
\text { Typical examples for each technology } \\
\text { Wired sensor network: bus netwo } \\
\text { Wireless sensor networks: short } \mathrm{r} \\
\text { Mesh networking: Zigbee, Z-Wa } \\
\text { Indoor localisation: proximity ser } \\
\text { Web of Things (WoT): REST, SO } \\
\text { Service Oriented Architecture (S } \\
\text { Cloud computing: XaaS, media c } \\
\text { Reactive programming: Actor mo } \\
\text { Human Computer Interaction (HC } \\
\text { Activity recognition: sensor patte } \\
\text { Identity management (IdM): fede } \\
\text { Artificial Intelligence (AI): Baye } \\
\text { Recommendation methods: conte } \\
\text { Semantics: Ontologies, SensorM }\end{array}$ & $\begin{array}{l}\text { isted above: } \\
\text { - CEBus/KNX } \\
\text { nge - Zigbee/Z- } \\
\text { BLE, 6LoWP } \\
\text { ing, radio finge } \\
\text { P, WS-*, HTT } \\
\text { A): gateway, O } \\
\text { ud, Fog compu } \\
\text { lels, Dataflow m } \\
\text { I): UI, BCI, ges } \\
\text { a matching, affe } \\
\text { ated identity, us } \\
\text { an network, AN } \\
\text { t based and coll } \\
\text { reasoning algo }\end{array}$ & $\begin{array}{l}\text { LonWorks, pov } \\
\text { Jave/BLE, cellı } \\
\text { N, etc. } \\
\text { rinting, space } \\
\text { CoAP, etc. } \\
\text { Gi, middleware } \\
\text { ng, etc. } \\
\text { dels, etc. } \\
\text { re recognition, } \\
\text { tive computing } \\
\text {-centric model } \\
\text { J, deep learning } \\
\text { borative filterin } \\
\text { thm, etc. }\end{array}$ & $\begin{array}{l}\text { er-line commu } \\
\text { ar networks-3c } \\
\text { acking, etc. } \\
\text { software agen } \\
\text { sability, UX, } \\
\text { behaviour prec } \\
\text { trust manager } \\
\text { etc. } \\
\text {, etc. }\end{array}$ & $\begin{array}{l}\text { ication - X1 } \\
\text { /LTE, TV w1 } \\
\text { s, discovery } \\
\text { c. } \\
\text { ction, etc. } \\
\text { ent, etc. }\end{array}$ & $\begin{array}{l}\mathrm{KNX} / \mathrm{Inste} \\
\text { ite space, ca } \\
\text { ervices, etc }\end{array}$ & $\begin{array}{l}\text { n, Ethernet, et } \\
\text { pillary network }\end{array}$ & etc. \\
\hline
\end{tabular}




\section{Challenges}

Research corporations around the world have been heavily promoting IoT and SH by predicting the huge potential of businesses in almost every market. Governments and institutions are investing immense amounts of resources to put the technologies in place for delivering IoT and SH services to the general public. However, there are still challenges that need to be addressed prior to a full SH and IoT implementation.

\subsection{Standardisation}

Although SH systems are domestic systems with most connected devices, such as appliances or sensors, locally installed. Mobile devices for instance smart phones, tablets and wearables travel from home to home, countries to countries. International standards must be established to govern from hardware interface, to communication protocols, to ontology description language, to semantic rules, to middleware. Standards developing organisations all around the world are proposing international standards for IoT and $\mathrm{SH}$, examples are OneM2 $\mathrm{M}^{16}$ from Europe and Asia, and IEEE P2413 ${ }^{17}$, etc. The initial release of the OneM2M specifications has just been announced recently and the first draft of P2413 is expected to be released in early 2016, so industry will still take some time to adopt the standards and produce consumer products.

\subsection{Security and Privacy for Smart Homes}

Enough examples of security and privacy violation have demonstrated the vulnerability of the existing Internet. IoT actually expands the Internet to a much wider scale which represents an even higher degree of risk. As we have described in the previous sections, new threats will become obvious when heterogeneous technologies are connected together. Research on security and privacy in a new dimension to include end to end system protection as a whole must be considered.

\subsection{UI for Pervasive Computing}

There is numerous research on the underlying IoT technologies, but the corresponding technological advancement on UI development is catching comparatively less attention. HCI has been providing concepts and theories for computers to interact with humans, and most of them are targeted to single computer interaction. Ubiquitous Computing or Pervasive Computing creates a new working relationship between humans and computers, both the quantity and the quality of computers are much different from the previous generation. Interaction with Pervasive Computing becomes a new experience thus new research for novel UI design is needed.

\subsection{Internet of People}

With the help of IoT, the Internet is expanding at an unprecedented scale connecting people all over the globe and even outside the globe (e.g. Interplanetary Web [86]). Internet connectivity has become an integral part of our daily life, especially the Millennial generation. IoT pushes the Internet connectivity to a new level that people are connected no matter they like it or not. The Executive Chairman of Google, Eric Schmidt, predicted that the Internet will disappear since nobody will notice the existence of the connection in the IoT world. Recent research is focusing on Internet of People based on IoT technologies since this is a new challenge for humans to interact with so many Things at the same time. Miranda et. al [87] realised the importance of humans with technologies and proposed a reference architecture for IoT developers and researchers to consider the relationship between humans and IoT systems. The ultimate goal of the Internet of People may come from a transition of the disappearing of computers (or pervasive computing) to the disappearing of Internet to the disappearing of interface and finally Humans and Things are seamlessly connected in a natural way.

\footnotetext{
${ }^{16} \mathrm{http}: / /$ onem2m.org/

${ }^{17} \mathrm{http}: / /$ standards.ieee.org/develop/project/2413.html
} 


\section{Conclusions and Future Work}

This paper has recommended a list of major requirements for building SH system. The seven major requirements proposed have been based on a taxonomy of architectures and technologies adopted in previous research. Meeting these requirements does not equate to a system that everybody will use, but it provides a common platform for building stronger SH applications. SH adoption rate is still low because there is no incentive for users to upgrade from ordinary homes to SHs, the remote controlling of heaters from offices is a hype rather than a necessity. However, real benefits are seen from saving energy through smart automation, from remote health monitoring for the elderly through tele-care services, and from controlling appliances for disabled persons through gesture interface or BCI.

User Interfaces tailor-made for intelligent homes are critical in a Human-centric technology like SH. Although HCI has been researched for decades with promising results to interact humans with single computers, a new paradigm for humans interacting with large number of invisible computers at home is a new and evolving topic.

\section{References}

[1] K. Ashton, That 'Internet of Things' Thing, RFiD Journal 22 (7) (2009) 97-114.

[2] D. Evans, The Internet of Things: How the Next Evolution of the Internet is Changing Everything, Tech. rep., CISCO white paper (2011). URL https://www.cisco.com/web/about/ac79/docs/innov/IoT_IBSG_0411FINAL.pdf

[3] J. Holler, V. Tsiatsis, C. Mulligan, S. Avesand, S. Karnouskos, D. Boyle, From Machine-to-Machine to the Internet of Things: Introduction to a New Age of Intelligence, Elsevier Science, 2014, Ch. 2.

[4] M. Weiser, The Computer for the 21st Century, Scientific american 265 (3) (1991) 94-104.

[5] E. Lee, Cyber Physical Systems: Design Challenges, in: Object Oriented Real-Time Distributed Computing (ISORC), 11th IEEE International Symposium on, 2008, pp. 363-369. doi:10.1109/ISORC. 2008.25.

[6] D. Guinard, What the Internet of Things Will Mean for the Smart Grid (June 2011). URL http://smartgrid.ieee.org/june-2011/95-what-the-internet-of-things-will-mean-for-the-smart-grid

[7] R. Drath, A. Horch, Industrie 4.0: Hit or Hype? [Industry Forum], Industrial Electronics Magazine, IEEE 8 (2) (2014) $56-58$. doi:10.1109/MIE. 2014.2312079.

[8] P. C. Evans, M. Annunziata, Industrial Internet: Pushing the Boundaries of Minds and Machines, Tech. rep., General Electric (2012). URL http://www.ge.com/sites/default/files/Industrial_Internet.pdf

[9] K. Gotkin, When Computers Were Amateur, Annals of the History of Computing, IEEE 36 (2) (2014) 4-14. doi : 10.1109/MAHC. 2014.32.

[10] J. Abbate, Getting small: a short history of the personal computer, in: Proceedings of the IEEE, Vol. 87, No.9, 1999, pp. 1695-1698. doi:10.1109/5.784256.

[11] B. Koyuncu, PC remote control of appliances by using telephone lines, Consumer Electronics, IEEE Transactions on 41 (1) (1995) $201-209$. doi: $10.1109 / 30.370328$.

[12] J. Greichen, Value based home automation for todays' market, Consumer Electronics, IEEE Transactions on 38 (3) (1992) XXXIV-XXXVIII. doi:10.1109/30.156666.

[13] C. Withanage, R. Ashok, C. Yuen, K. Otto, A comparison of the popular home automation technologies, in: Innovative Smart Grid Technologies - Asia (ISGT Asia), IEEE, 2014, pp. 600-605. doi:10.1109/ISGT-Asia.2014.6873860.

[14] J. Sachs, Capillary networks - a smart way to get things connected, Tech. rep., Ericsson Review (9 2014). URL http://www.ericsson.com/news/140908-capillary-networks_244099436_c

[15] L. Bedogni, A. Trotta, M. Di Felice, L. Bononi, Machine-to-Machine Communication over TV White Spaces for Smart Metering Applications, in: Computer Communications and Networks (ICCCN), 22nd International Conference on, 2013, pp. 1-7. doi: 10.1109/ICCCN. 2013.6614149.

[16] W. Webb, Standard's net gains [Communications Emerging Standards], Engineering Technology 8 (5) (2013) 76-78. doi:10.1049/et.2013.0512.

[17] T. Yamazaki, Beyond the Smart Home, in: Hybrid Information Technology, ICHIT '06. International Conference on, Vol. 2, 2006, pp. 350355. doi:10.1109/ICHIT.2006.253633.

[18] T. Perumal, A. R. Ramli, C. Y. Leong, S. Mansor, K. Samsudin, Interoperability among Heterogeneous Systems in Smart Home Environment, in: Signal Image Technology and Internet Based Systems, SITIS '08. IEEE International Conference on, 2008, pp. 177-186. doi:10.1109/SITIS.2008.94.

[19] L. Xuemei, X. Gang, Service Oriented Framework for Modern Home Appliances, in: Computing, Communication, Control, and Management, CCCM '08. ISECS International Colloquium on, Vol. 1, 2008, pp. 700-703. doi:10.1109/CCCM. 2008. 386.

[20] S. Hachem, T. Teixeira, V. Issarny, Ontologies for the Internet of Things, in: Proceedings of the 8th Middleware Doctoral Symposium, MDS '11, ACM, New York, NY, USA, 2011, pp. 3:1-3:6. doi:10.1145/2093190. 2093193.

[21] E. Rubio-Drosdov, D. Díaz-Sánchez, P. Arias-Cabarcos, F. Almenárez, A. Marn, Towards a seamless human interaction in IoT, in: Consumer Electronics (ISCE), 2015 IEEE 19th International Symposium on, 2015, pp. 1-2. doi:10.1109/ISCE. 2015.7177781.

[22] D. Guinard, V. Trifa, E. Wilde, Architecting a Mashable Open World Wide Web of Things, Technical Report 663, Institute for Pervasive Computing, ETH Zurich (Feb 2010). URL http://www.vs.inf.ethz.ch/publ/papers/WoT.pdf

[23] C. Maternaghan, K. Turner, Programming home care, in: Pervasive Computing Technologies for Healthcare (PervasiveHealth), 5th International Conference on, 2011, pp. 485-491. 
[24] D. Díaz-Sánchez, F. Almenárez, A. Marin, D. Proserpio, P. Arias Cabarcos, Media cloud: an open cloud computing middleware for content management, Consumer Electronics, IEEE Transactions on 57 (2) (2011) 970-978. doi : 10.1109/TCE. 2011.5955247.

[25] M. Tan, X. Su, Media cloud: When media revolution meets rise of cloud computing, in: Service Oriented System Engineering (SOSE), IEEE 6th International Symposium on, 2011, pp. 251-261. doi:10.1109/SOSE. 2011.6139114.

[26] V. D. K. Mai, Y. Kim, Using DLNA cloud for sharing multimedia contents beyond home networks, in: Advanced Communication Technology (ICACT), 16th International Conference on, 2014, pp. 54-57. doi:10.1109/ICACT . 2014.6778921.

[27] D. Díaz-Sánchez, F. Almenárez, A. Marín, R. S. Guerrero, P. Arias, Media Gateway: bringing privacy to private multimedia cloud connections, Telecommunication Systems 55 (2) (2014) 315-330. doi : 10.1007/s11235-013-9783-1.

[28] F. Bonomi, R. Milito, J. Zhu, S. Addepalli, Fog Computing and Its Role in the Internet of Things, in: Proceedings of the First Edition of the MCC Workshop on Mobile Cloud Computing, MCC'12, ACM, New York, NY, USA, 2012, pp. 13-16. doi : 10.1145/2342509.2342513.

[29] I. Stojmenovic, Fog computing: A cloud to the ground support for smart things and machine-to-machine networks, in: Telecommunication Networks and Applications Conference (ATNAC), Australasian, 2014, pp. 117-122. doi:10.1109/ATNAC. 2014.7020884.

[30] M. Soliman, T. Abiodun, T. Hamouda, J. Zhou, C.-H. Lung, Smart Home: Integrating Internet of Things with Web Services and Cloud Computing, in: Cloud Computing Technology and Science (CloudCom), IEEE 5th International Conference on, Vol. 2, 2013, pp. 317-320. doi:10.1109/CloudCom.2013.155

[31] K. I. Hwang, Designing robust ZigBee networks with Enhanced Self-Configuration, in: Consumer Electronics, ICCE '09. Digest of Technical Papers International Conference on, 2009, pp. 1-2. doi:10.1109/ICCE.2009.5012234.

[32] F. Cuomo, T. Melodia, I. Akyildiz, Distributed self-healing and variable topology optimization algorithms for QoS provisioning in scatternets, Selected Areas in Communications, IEEE Journal on 22 (7) (2004) 1220-1236. doi:10.1109/JSAC. 2004.829341.

[33] K.-H. Chang, Bluetooth: a viable solution for IoT? [Industry Perspectives], Wireless Communications, IEEE 21 (6) (2014) 6-7. doi:10.1109/MWC. 2014.7000963.

[34] G. Leeb, R. Posta, G. Schildt, M. Ochensthaler, D. Dietrich, A configuration tool for HomeNet, Consumer Electronics, IEEE Transactions on 42 (3) (1996) 387-394. doi:10.1109/30.536135.

[35] S.-Y. Chen, Y.-F. Chang, The Computer-Aided Design Software for Smart Home Device Based on Cloud Computing Service, in: Software Engineering (WCSE), Second World Congress on, Vol. 1, 2010, pp. 273-278. doi:10.1109/WCSE. 2010.21.

[36] G. Aloisio, D. Conte, C. Elefante, G. Marra, G. Mastrantonio, G. Quarta, Globus Monitoring and Discovery Service and SensorML for Grid Sensor Networks, in: Enabling Technologies: Infrastructure for Collaborative Enterprises, WETICE '06. 15th IEEE International Workshops on, 2006, pp. 201-206. doi:10.1109/WETICE. 2006.44.

[37] S. Brown, C. J. Sreenan, Software Updating in Wireless Sensor Networks: A Survey and Lacunae, Journal of Sensor and Actuator Networks 2 (4) (2013) 717-760. doi:10.3390/jsan2040717.

[38] H.-Y. Huang, W.-C. Teng, S.-L. Chung, Smart home at a finger tip: OSGi-based MyHome, in: Systems, Man and Cybernetics. IEEE International Conference on, 2009, pp. 4467-4472. doi:10.1109/ICSMC. 2009.5346916.

[39] D. Díaz-Sánchez, R. Sherratt, P. Arias-Cabarcos, F. Almenárez, A. Marín, Enabling Actor Model for Crowd Sensing and IoT, in: Consumer Electronics (ISCE), 2015 IEEE 19th International Symposium on, 2015, pp. 1-2. doi : 10.1109/ISCE. 2015.7177779.

[40] B. Schilit, N. Adams, R. Want, Context-Aware Computing Applications, in: Mobile Computing Systems and Applications. First Workshop on, 1994, pp. 85-90. doi:10.1109/WMCSA.1994.16.

[41] T. Choudhury, S. Consolvo, B. Harrison, J. Hightower, A. Lamarca, L. Legrand, A. Rahimi, A. Rea, G. Bordello, B. Hemingway, P. Klasnja, K. Koscher, J. A. Landay, J. Lester, D. Wyatt, D. Haehnel, The mobile sensing platform: An embedded activity recognition system, Pervasive Computing, IEEE 7 (2) (2008) 32-41. doi:10.1109/MPRV.2008.39.

[42] T. Yamazaki, The ubiquitous home, International Journal of Smart Home 1 (1) (2007) 17-22.

[43] Y.-G. Ha, Y.-C. Byun, A Ubiquitous Homecare Service System Using a Wearable User Interface Device, in: Computer and Information Science (ICIS), IEEE/ACIS 11th International Conference on, 2012, pp. 649-650. doi:10.1109/ICIS.2012.22.

[44] T. Rahman, A. T. Adams, M. Zhang, E. Cherry, T. Choudhury, Bodybeat: Eavesdropping on our body using a wearable microphone, GetMobile: Mobile Computing and Communications 19 (1) (2015) 14-17. doi:10.1145/2786984.2786989.

[45] C. S. S. Tan, J. Schöning, K. Luyten, K. Coninx, Informing intelligent user interfaces by inferring affective states from body postures in ubiquitous computing environments, in: Proceedings of the International Conference on Intelligent User Interfaces, IUI '13, ACM, New York, NY, USA, 2013, pp. 235-246. doi:10.1145/2449396.2449427.

[46] I. Bisio, A. Delfino, F. Lavagetto, M. Marchese, A. Sciarrone, Gender-driven emotion recognition through speech signals for ambient intelligence applications, Emerging Topics in Computing, IEEE Transactions on 1 (2) (2013) 244-257. doi : 10.1109/TETC.2013.2274797.

[47] J. Corn, User Unfriendly: Consumer Struggles with Personal Technologies, from Clocks and Sewing Machines to Cars and Computers, The John Hopkins University Press, 2011.

[48] S. Moeller, K.-P. Engelbrecht, S. Hillmann, P. Ehrenbrink, New ITG Guideline for the Usability Evaluation of Smart Home Environments, in: Speech Communication; 11. ITG Symposium; Proceedings of, 2014, pp. 1-4.

[49] F. Vazquez, W. Kastner, Detecting user dissatisfaction in ambient intelligence environments, in: Emerging Technologies Factory Automation (ETFA), IEEE 17th Conference on, 2012, pp. 1-4. doi:10.1109/ETFA. 2012.6489748.

[50] G. Epelde, X. Valencia, J. Abascal, U. Diaz, I. Zinnikus, C. Husodo-Schulz, TV as a human interface for Ambient Intelligence environments, in: Multimedia and Expo (ICME), IEEE International Conference on, 2011, pp. 1-6. doi : 10.1109/ICME. 2011.6012186.

[51] M. Hossain, P. Atrey, A. Saddik, Smart mirror for ambient home environment, in: Intelligent Environments, IE 07., 3rd IET International Conference on, 2007, pp. 589-596.

[52] K. Tokuda, S. Matsumoto, M. Nakamura, Implementing personal home controllers on smartphones for service-oriented home network, in: Wireless and Mobile Computing, Networking and Communications (WiMob), IEEE 8th International Conference on, 2012, pp. 769-776. doi:10.1109/WiMOB.2012.6379162.

[53] G. Coelho, A. Serralheiro, J. Netti, Microphone Array Front-End Interface for Home Automation, in: Hands-Free Speech Communication and Microphone Arrays, 2008, pp. 184-187. doi:10.1109/HSCMA.2008.4538717.

[54] A. Ullah, M. Islam, S. Aktar, S. Hossain, Remote-touch: Augmented reality based marker tracking for smart home control, in: Computer and 
Information Technology (ICCIT), 15th International Conference on, 2012, pp. 473-477. doi:10.1109/ICCITechn. 2012.6509774.

[55] Z. Bien, K.-H. Park, J.-W. Jung, J.-H. Do, Intention reading is essential in human-friendly interfaces for the elderly and the handicapped, Industrial Electronics, IEEE Transactions on 52 (6) (2005) 1500-1505. doi:10.1109/TIE. 2005.858734.

[56] A. Grguric, M. Mosmondor, M. Kusek, C. Stocklow, D. Salvi, Introducing gesture interaction in the Ambient Assisted Living platform universaal, in: Telecommunications (ConTEL), 12th International Conference on, 2013, pp. 215-222.

[57] W. T. Lee, H. Nisar, A. Malik, K. H. Yea, A brain computer interface for smart home control, in: Consumer Electronics (ISCE), IEEE 17th International Symposium on, 2013, pp. 35-36. doi:10.1109/ISCE. 2013.6570240.

[58] A. Kamilaris, A. Pitsillides, Social networking of the Smart Home, in: Personal Indoor and Mobile Radio Communications (PIMRC), IEEE 21st International Symposium on, 2010, pp. 2632-2637. doi : 10.1109/PIMRC. 2010.5671783.

[59] R. Cristian, R. Virginica, R. Silvana, G. Carina, Usability and User Experience: What Should We Care About?, International Journal of Information Technologies and Systems Approach (IJTSA) 8 (2) (2015) 1-12. doi:10.4018/IJITSA. 2015070101.

[60] M. Kurosu, A. Hashizume, Describing Experiences in Different Modes of Behavior: GOB, POB and SOB, International Journal of Affective Engineering 12 (2) (2013) 291-298. doi:10.5057/ijae.12.291.

[61] D. Vavilov, A. Melezhik, I. Platonov, Reference model for Smart Home user behavior analysis software module, in: Consumer Electronics Berlin (ICCE-Berlin), IEEE Fourth International Conference on, 2014, pp. 3-6. doi:10.1109/ICCE-Berlin. 2014.7034262.

[62] D. Fortin-Simard, J. Bilodeau, K. Bouchard, S. Gaboury, B. Bouchard, A. Bouzouane, Exploiting Passive RFID Technology for Activity Recognition in Smart Homes, Intelligent Systems, IEEE 30 (Issue 4) (2015) 7-15. doi : 10.1109/MIS.2015.18.

[63] D. Mitchell, P. J. Morrow, C. D. Nugent, A sensor and video based ontology for activity recognition in smart environments, in: Engineering in Medicine and Biology Society (EMBC), 36th Annual International Conference of the IEEE, 2014, pp. 5932-5935. doi:10.1109/EMBC.2014.6944979.

[64] Z. Huiru, W. Haiying, N. Black, Human Activity Detection in Smart Home Environment with Self-Adaptive Neural Networks, in: Networking, Sensing and Control. ICNSC'08. IEEE International Conference on, 2008, pp. 1505-1510. doi:10.1109/ICNSC. 2008.4525459.

[65] J. Cu, R. Cabredo, G. Cu, R. Legaspi, P. S. Inventado, R. Trogo, M. T. Suarez, The TALA Empathic Space: Integrating Affect and Activity Recognition into a Smart Space, in: Human-Centric Computing (HumanCom), 3rd International Conference on, 2010, pp. 1-6. doi: 10.1109/HUMANCOM. 2010.5563342.

[66] S. Azzi, C. Dallaire, A. Bouzouane, B. Bouchard, S. Giroux, Human activity recognition in big data smart home context, in: Big Data (Big Data), IEEE International Conference on, 2014, pp. 1-8. doi:10.1109/BigData.2014.7004406.

[67] F. Hongqing, H. Chen, Recognizing human activity in smart home using deep learning algorithm, in: Control Conference (CCC), 33rd Chinese, 2014, pp. 4716-4720. doi:10.1109/ChiCC. 2014.6895735

[68] K. Islam, W. Shen, X. Wang, Security and privacy considerations for Wireless Sensor Networks in smart home environments, in: Computer Supported Cooperative Work in Design (CSCWD), IEEE 16th International Conference on, 2012, pp. 626-633. doi:10.1109/CSCWD. 2012.6221884.

[69] I. Sanchez, R. Satta, I. Fovino, G. Baldini, G. Steri, D. Shaw, A. Ciardulli, Privacy leakages in Smart Home wireless technologies, in: Security Technology (ICCST), International Carnahan Conference on, 2014, pp. 1-6. doi:10.1109/CCST . 2014.6986977.

[70] P. Fremantle, B. Aziz, J. Kopecky, P. Scott, Federated Identity and Access Management for the Internet of Things, in: Secure Internet of Things (SIoT), International Workshop on, 2014, pp. 10-17. doi:10.1109/SIoT.2014.8.

[71] Organization for the Advancement of Structured Information Standards, Security Assertion Markup Language (SAML) v2.0 (2005). URL http://www.bibsonomy.org/bibtex/27067051e153c2951a38faf137be1bdd9/direx

[72] E. Maler, D. Reed, The Venn of Identity: Options and Issues in Federated Identity Management, IEEE Security \& Privacy 6 (2) (2008) 16-23. doi:10.1109/MSP.2008.50.

[73] P. Arias Cabarcos, F. Almenárez Mendoza, A. Marn-López, D. Díaz-Sánchez, Enabling SAML for Dynamic Identity Federation Management, in: Wireless and Mobile Networking, Vol. 308 of IFIP Advances in Information and Communication Technology, Springer Berlin Heidelberg, 2009, pp. 173-184. doi:10.1007/978-3-642-03841-9_16.

[74] F. A. Mendoza, A. M. López, D. Díaz-Sánchez, A. Cortés, C. Campo, C. García-Rubio, A Trust-based Middleware for Providing Security to Ad-Hoc Peer-to-Peer Applications, in: Sixth Annual IEEE International Conference on Pervasive Computing and Communications (PerCom), 17-21 March, 2008, pp. 531-536. doi:10.1109/PERCOM. 2008.95.

[75] Y. Wang, J. Vassileva, Trust and reputation model in peer-to-peer networks, in: Peer-to-Peer Computing, (P2P'03). Proceedings. Third International Conference on, 2003, pp. 150-157. doi:10.1109/PTP. 2003.1231515.

[76] E. Bertino, L. Khan, R. Sandhu, B. Thuraisingham, Secure knowledge management: confidentiality, trust, and privacy, Systems, Man and Cybernetics, Part A: Systems and Humans, IEEE Transactions on 36 (3) (2006) 429-438. doi : 10.1109/TSMCA . 2006. 871796.

[77] D. Díaz-Sánchez, A. M. López, F. A. Mendoza, C. Campo, A. Cortés, C. García-Rubio, Trust Negotiation Protocol Support for Secure Mobile Network Service Deployment, in: Wireless and Mobile Networking, IFIP Joint Conference on Mobile and Wireless Communications Networks (MWCN'08) and Personal Wireless Communications (PWC'08), Toulouse, France, September 30 - October 2, 2008 , pp. $271-282$. doi: 10.1007/978-0-387-84839-6_22.

[78] P. A. Cabarcos, F. A. Mendoza, F. G. Mármol, A. Marín, To Federate or Not To Federate: A Reputation-Based Mechanism to Dynamize Cooperation in Identity Management, Wireless Personal Communications 75 (3) (2014) 1769-1786. doi:10.1007/s11277-013-1338-y.

[79] W. G. Ryan, Privacy and freedom: Alan F. Westin Atheneum Publishers, Business Horizons 10 (4) (1967) 106-106.

[80] C.-H. Liang, W.-S. Hung, M.-C. Hsieh, C.-M. Wu, C.-H. Luo, A Multi-agent Based Architecture for an Assistive User Interface of Intelligent Home Environment Control, in: Intelligent Systems Design and Applications. ISDA '08. Eighth International Conference on, Vol. 1, 2008, pp. 335-338. doi:10.1109/ISDA.2008.320.

[81] M.-O. Pahl, A. Muller, G. Carle, C. Niedermeier, M. Schuster, Knowledge-based middleware for future home networks, in: Wireless Days (WD), 2nd IFIP, 2009, pp. 1-6. doi:10.1109/WD. 2009.5449684.

[82] J. Chin, V. Callaghan, G. Clarke, Soft-appliances: A vision for user created networked appliances in digital homes, Journal of Ambient Intelligence and Smart Environments 1 (1) (2009) 69-75. doi:10.3233/AIS-2009-0010.

[83] C. Fabbricatore, H. Boley, A. Karduck, Machine learning for resource management in smart environments, in: Digital Ecosystems 
Technologies (DEST), 6th IEEE International Conference on, 2012, pp. 1-6. doi:10.1109/DEST . 2012.6227910.

[84] J. Chen, J. Ma, N. Zhong, Y. Yao, J. Liu, R. Huang, W. Li, Z. Huang, Y. Gao, J. Cao, WaaS: Wisdom as a Service, Intelligent Systems, IEEE 29 (6) (2014) 40-47. doi:10.1109/MIS. 2014.19.

[85] G. Adomavicius, A. Tuzhilin, Toward the next generation of recommender systems: a survey of the state-of-the-art and possible extensions, Knowledge and Data Engineering, IEEE Transactions on 17 (6) (2005) 734-749. doi:10.1109/TKDE. 2005.99.

[86] V. Pendyala, S. Shim, C. Bussler, The web that extends beyond the world, Computer 48 (5) (2015) 18-25. doi:10.1109/MC.2015.150.

[87] J. Miranda, N. Makitalo, J. Garcia-Alonso, J. Berrocal, T. Mikkonen, C. Canal, J. Murillo, From the Internet of Things to the Internet of People, Internet Computing, IEEE 19 (2) (2015) 40-47. doi:10.1109/MIC.2015.24. 